\title{
Key Dynamic Parameters that Influence Ride Quality of Passenger Transportation Systems
}

\author{
Thomas Ehrl ${ }^{1}$, Rory Smith ${ }^{2}$, Stefan Kaczmarczyk ${ }^{3}$ \\ ${ }^{1}$ Thyssenkrupp Elevator Innovation GmbH, Germany, e-mail: thomas.ehrl@northampton.ac.uk \\ ${ }^{2}$ ThyssenKrupp Americas Inc., United States of America, email: rory.smith@thyssenkrupp.com \\ ${ }^{3}$ Faculty of Arts, Science and Technology, The University of Northampton, United Kingdom, e-mail: \\ stefan.kaczmarczyk@northampton.ac.uk
}

Keywords: Lift, Escalator, Noise and Vibration, Design, Components, Dynamic Interactions

\begin{abstract}
Ride quality of a Passenger Transportation System (PTS) is a measure of the comfort level experienced by passengers and is intimately associated with their subjective perception and sensitivity to motion and sound. This measure is affected by the noise and vibration of an operating system. On the other hand, ride quality is the measure of the PTS product quality. Ride quality of passenger transportation systems is critical for a PTS manufacturer to determine the subjective and objective quality of the system. This is especially important in high rise (high end) systems. The paper investigates the dynamic interactions that might occur between the PTS system components and their influence on ride quality.
\end{abstract}

\section{INTRODUCTION}

All humans are different and so is the perceived ride performance of a Passenger Transportation System (PTS), either in a vertical direction using a lift or in a horizontal direction using a moving walkway or an escalator. However, ride quality perceptions in the horizontal direction and the vertical direction, are not the same. The acceptance levels of a given PTS assessed by a user group vary from user to user and typically show wide differences [1].

A PTS contains of a large number of components which interact with each other. These components or sub-systems influence the ride quality of the system [2] and it is in the interest of the PTS manufacturer to install and run systems that make the ride as smooth as possible. The issues of how these components dynamically influence the PTS and how humans respond to it are discussed in this paper. Based on the results presented in the paper, some measures to improve the ride performance are suggested.

\section{DEFINITION OF RIDE QUALITY}

Ride quality of a PTS is a measure of the comfort level experienced by passengers and is intimately associated with their subjective perception and sensitivity to motion and to sound. In the case of a lift system the car motion and noise levels may compromise ride quality [3]. Ride quality affected by motion and sound can be quantified by using the following parameters:

- Horizontal vibrations. The horizontal vibrations are defined as the lateral motions (in the $\mathrm{x}$ - or y-direction, respectively, see Fig. 1). These motions are characterized in terms of the frequency (measured in $\mathrm{Hz}$ ) and the amplitude (represented by the magnitude of acceleration and measured in $\left.\mathrm{m} / \mathrm{s}^{2}\right)$. The acceleration amplitude can also be measured in milli-g (1 milli-g $\left.=0.00981 \mathrm{~m} / \mathrm{s}^{2}\right)$. 


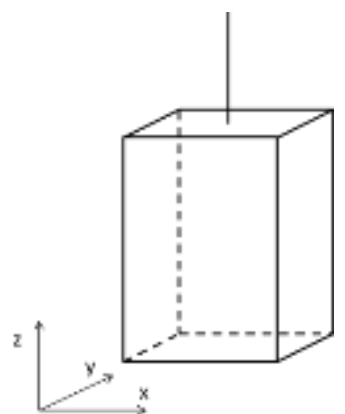

Figure 1. Arrangement of the $x-$, $y$ - and z-axis.

- Vertical vibrations (with the amplitude measured in $\mathrm{m} / \mathrm{s}^{2}$ and the frequency measured in $\mathrm{Hz}$ ). The vertical vibrations represent motions in the direction of the z-axis (see Fig. 1). A typical cause of this vertical vibration is the elasticity of the suspension mean, such as a steel-wire rope.

- Acceleration of the transportation (overall) motion (measured in $\mathrm{m} / \mathrm{s}^{2}$ ). This is the rate of change of speed in an upwards or downwards direction (direction of travel). Positive acceleration indicates the increase of speed. Negative acceleration (deceleration) indicates the decrease of speed.

- Jerk (measured in $\mathrm{m} / \mathrm{s}^{3}$ ) is the time-derivative of acceleration (or the rate of change of acceleration).

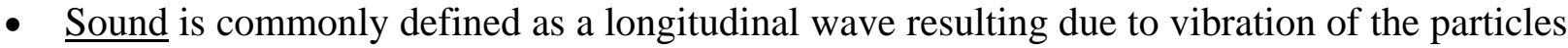
in the medium (e.g. air/ gas, liquids, solids) through which the wave is moving. The sound wave causes the local pressure deviation from the ambient pressure which is referred to as the sound pressure (measurements are in $\mathrm{Pa}$ ). The sound pressure level is quantified as the logarithmic measure of the effective pressure of a sound relative to a reference value (measured in decibels $\mathrm{dB}$ or $\mathrm{A}$-weighted decibels $\mathrm{dB}(\mathrm{A})$ applied to account for the relative loudness perception of the human ear).

- Tympanic pressure (measured in $\mathrm{Pa}$ ) representing the effect of the dynamic change of ear pressure in the middle ear causing discomfort.

The acceptance levels of these aspects not only vary from human to human, but are also different in terms of product range (e.g. low-cost low-rise - middle segment - high-speed/high rise). Therefore, case-specific pass/fail or assessment criteria for PTS are put aside for the purposes of this paper.

\section{IMPACT ON HUMAN DISCOMFORT}

The response of the complex active structure of a human body to the multiple facets of ride quality is unique from human to human. The actions of noise and vibration upon a human body can create various subjective effects and at the end may cause human discomfort.

Humans are different in terms of body mass, portion of muscles, physical ability or overall condition [4,5]. Thus, the sensitivity to external stimulations is again different from person to person. If it comes to extremes, in the case of very poor ride quality with human over-sensitivity, vibrations can cause stress effects to the following physiological areas: to the cardio-vascular system, nervous system, muscles, and respiratory system, respectively.

It is common practice in the lift industry to maintain critical quantities that affect ride quality within certain limits to ensure good ride quality. Those limits can be defined as follows.

- Humans are more sensitive to jerk rates than to acceleration, and jerk rates less than $1.0 \mathrm{~m} / \mathrm{s}^{3}$ are assessed as comfortable [2]. 
- High rise/high speed travel (e.g.: travel distances of $400 \mathrm{~m}$ and more) with a maximum speed greater than $7 \mathrm{~m} / \mathrm{s}$ usually causes an uncomfortable trip in a PTS due aerodynamic effects (causing higher levels of noise and vibration in the car).

- Acceleration rates within the range of $0.8-1.6 \mathrm{~m} / \mathrm{s}^{2}$ are acceptable, however, especially for high rise application these limits are disregarded due to transportation capacity requirements.

- Horizontal vibrations are typically specified below 25 milli-g (peak to peak in the $\mathrm{x}$ - and y-axis).

- Maintaining the maximum limit sound level of less than $55 \mathrm{~dB}(\mathrm{~A})$ is desired in the lift car.

In the system of 'human-machine/lift' a passenger interacts with the lift car structure. The passenger stands on the car floor, typically wearing shoes. These represent coupling or damping elements between the human body and the lift system (car). The effects of various damping characteristics of different types of shoes and the corresponding theoretical and experimental transmissibility have been studied and discussed in [6]. The dynamic response of the lift car can be influenced substantially by the characteristics of the passengers and their behaviour during the lift travel. The dynamic interactions between the lift components can be quantified in terms of their mass-damping-stiffness characteristics and count in the context of human-machine dynamic system.

\section{COMPONENTS THAT AFFECT RIDE QUALITY}

\subsection{Sources of vibrations}

The operation of lift systems is influenced by vibrations and associated vibroacoustic noise phenomena. These phenomena affect car ride quality and lead to high levels of dynamic stresses in the lift components. A good understanding and prediction of vibration and noise effects occurring in lift installations are essential for developing effective suppression and control strategies in order to design a system which satisfies ever demanding ride quality criteria.

Vibration sources affecting a lift car involve the car guiding system, suspension and compensating ropes and air flow $[7,8]$. The underlying causes of vibration are varied, including poorly aligned joints and imperfections of guide, eccentric pulleys and sheaves, systematic resonance in the electronic control system, and gear and motor generated vibrations $[9,10]$.

In high-rise applications elevators are subject to extreme loading conditions. High-rise buildings sway at low frequencies and large amplitudes due to adverse wind conditions and the load resulting from the building sway excites the lift system. This leads to large vibratory motions of lift ropes $[11,12]$. The taller a building, the higher the rated speeds of elevator systems are needed. The dynamic responses become more adverse as the speed increases. Torque ripple generated in the motor causes vertical vibrations of the car [13]. At high speeds guide rail deformations induce large lateral vibrations of the car [14]. Furthermore, large aerodynamic loadings due to the airflow around the car result in excessive noise and flow-induced vibrations of the car structure $[15,16]$.

Following on from the above it is evident that a variety of sources may affect the lift car and - if a passenger travels in the cabin - the passenger. The primary sources that affect car ride quality can be identified as follows:

- guiding system (faulty guide rails and joints),

- suspension and compensating ropes,

- air flow,

- imperfect pulleys and sheaves,

- building sway transmitted to elastic components of the lift system, such as ropes and cables. 


\subsection{Vibration frequency ranges}

Guide rails. The most essential elements affecting ride quality of a lift are guide rails and their installation condition. Due to the T-profile design and the manufacturing process, guiderails are never perfectly straight and are not rigid [2]. Guide rail irregularities (poorly aligned joints, bends and unevenness) introduce lateral excitation to the car during its travel. The range of excitation frequencies depends on the speed. The fundamental frequency of this excitation is given as

$$
\Omega=\frac{2 \pi}{\lambda} V \frac{\text { rad }}{s}\left(\text { or } f=\frac{\Omega}{2 \pi}=\frac{V}{\lambda} H z\right)
$$

where $\lambda$ represents the wavelength corresponding to the guide rail profile.

For example, in a standard arrangement the two lengths of guide rail are connected together with a rectangular cross section fishplate bridging the joint. The joint interface is the critical area as it is extremely difficult to arrange that the second moment of area $(I)$ of the fishplate - joint interface is the same as that of the guide rail. Thus, the bending stiffness ( $E I$, where $E$ is the Young's modulus) of the guiding system is non-uniform in respect of bending deflections. Therefore, during lift travel with a given speed of $V$, the frequency $f$ of excitation transmitted to the car frame can be calculated from Eq. 1 as

$$
f=\frac{V}{L}[H z]
$$

where $L$ is the rail length. Thus, using the standard rail length of $5 \mathrm{~m}$ and the speed range of $10-18$ $\mathrm{m} / \mathrm{s}$ this would yield the frequency range of $2-3.6 \mathrm{~Hz}$. On the other hand, taking into account the fact that the wavelength due to manufacturing bends are about $0.1 \mathrm{~m}$ [8] the frequencies calculated from equation (1) can be over $100 \mathrm{~Hz}(100-180 \mathrm{~Hz})$.

It should be noted that the nature of guide rail imperfections should be classified as nondeterministic. If the unevenness of guide rails is measured then the record for one rail will be different from that for another one. This characteristic is referred to as being random or stochastic [12]. Consequently, the response of a car - hoist rope system is also a random phenomenon.

Roller Guides. Roller guides are available in various types and configurations for all kinds of application, such as high speed traction lifts or low rise hydraulic systems. Under the consideration of stiffness and damping characteristic of these rotating or non-rotating components, it is obvious that they directly affect the vibration and thus the ride performance of a lift car.

Imperfections of rotating components such as traction sheave, diverter pulleys. Rotating components can only be made to its ideal round condition with huge manufacturing efforts. In consequence, the rotating components deviate from the geometrical dimensional data and an imbalance excites the system with a frequency of its rotational velocity.

Rope dynamics. Due to their flexibility, hoist (suspension) and compensating ropes are susceptible to vibrations. These vibrations are transmitted to a lift car which often results in a ride quality which is unacceptable [17]. Lift ropes can vibrate in the vertical (longitudinal) direction and lateral (horizontal) in-plane and out-of-plane directions (see Fig. 2). An important feature of a lift system is that the ropes are of time varying length. Furthermore, the number of passengers on board (load) changes. Consequently, the dynamic characteristics of the system vary during travel. In particular, the natural frequencies of the ropes vary slowly during the elevator car motion rendering the system non-stationary. An adverse situation arises when one of the slowly varying rope frequencies approaches near the frequency of a periodic excitation existing in the system. This results in a passage through external resonances. 


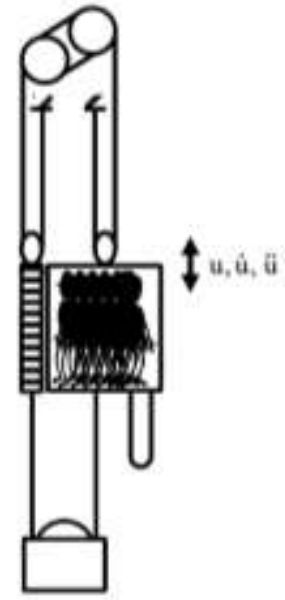

(a)

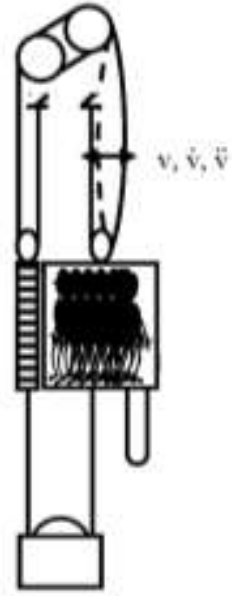

(b)

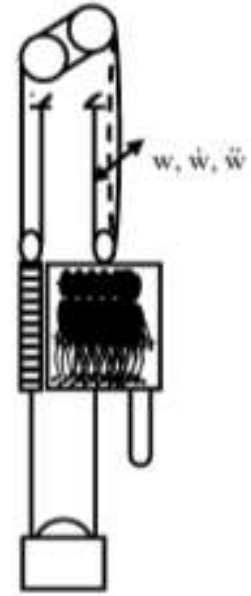

(c)

Figure 2. Longitudinal (a), lateral in-plane (b) and lateral out-of-plane (c) vibrations of elevator ropes [8]

Overall, during the system operation the range of resonance frequencies of the ropes may vary from below $1 \mathrm{~Hz}$ to over $100 \mathrm{~Hz}$. As far as the interactions between the ropes and the car are concerned, a lift will not vibrate throughout its travel at high amplitudes but will 'pass through' a resonant vibration at some particular stage in the travel. An important excitation source relevant to the ropecar assembly interactions is the low frequency building sway, typically below $0.5-0.1 \mathrm{~Hz}$.

\subsection{Noise sources and frequency band}

The human hearing range defines the band of frequencies that humans can hear and is usually specifies as $20 \mathrm{~Hz}$ to $20,000 \mathrm{~Hz}$, whereas there is substantial variation between individuals, especially at high frequencies. The sensitivity for noise also varies with frequency. However, the noticeable range of vibration for human beings is even below the minimum of hearable noise. A study to characterize the most important vibroacoustic energy sources and to identify the dominant paths corresponding to broadband $(100-500 \mathrm{~Hz})$ acoustic energy transmission to the car interior in high-rise lift installations has been carried out by Coffen et al. [18].

It has been identified that in high-rise lift installations cars are subject to structure-borne as well as to air-borne noise. Structure-borne noise is caused mainly by the vibration induced by the car roller guides - guide rail interaction and by the hoist rope - rope hitch interface. This structure-borne vibroacoustic energy is transmitted to the car interior through the car frame structure (and in particular by the uprights). The air-borne noise is generated by aerodynamic effects during the car travel. It includes shaft noise entering the car through the ventilation openings and the door seals. The air (flow)-induced vibrations of the car exterior panels generate noise that is transmitted to the car interior.

\section{DYNAMIC INTERACTIONS}

It is the physical effect of resonance that may cause issues with noise and vibration in PTSs. The primary external resonance arises when the frequency of external excitation becomes close to one of the natural frequencies of the system. The natural frequencies of a given component is associated with one of the many standing wave patterns by which that component could vibrate. Any component can be forced into vibration at one of its natural frequencies (harmonics) if another interconnected object 
acts upon it with one of those frequencies. With focus on the issue of resonance and its influence to the dynamics of lift cars the following case study is considered.

- The installation comprises a lift system with a car of rated speed $1.6 \mathrm{~m} / \mathrm{s}$ and travel height 23.6 $\mathrm{m}$ with multi-reeving roping arrangement.

- The car suffers from excessive vertical (z-axis) vibrations (peak-to-peak acceleration amplitude of over $58 \mathrm{mg}$; please see the time measurement record in Fig. 3 and the corresponding Fast Fourier Transform (FFT) frequency spectrum in Fig. 4)

- The fundamental frequency of the response is about $3.25 \mathrm{~Hz}$ which is close to the rotational speed in the diverter pulley system determined as $3.18 \mathrm{~Hz}$.

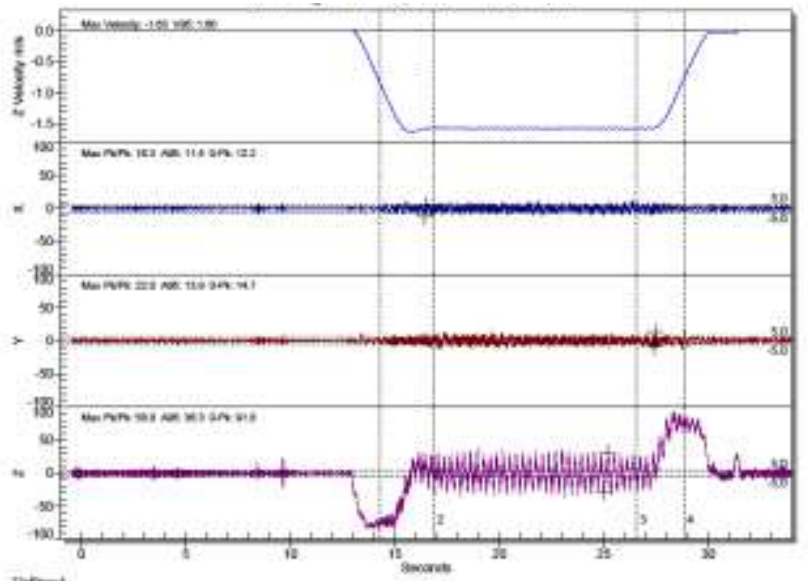

Figure 3. Time measurement record

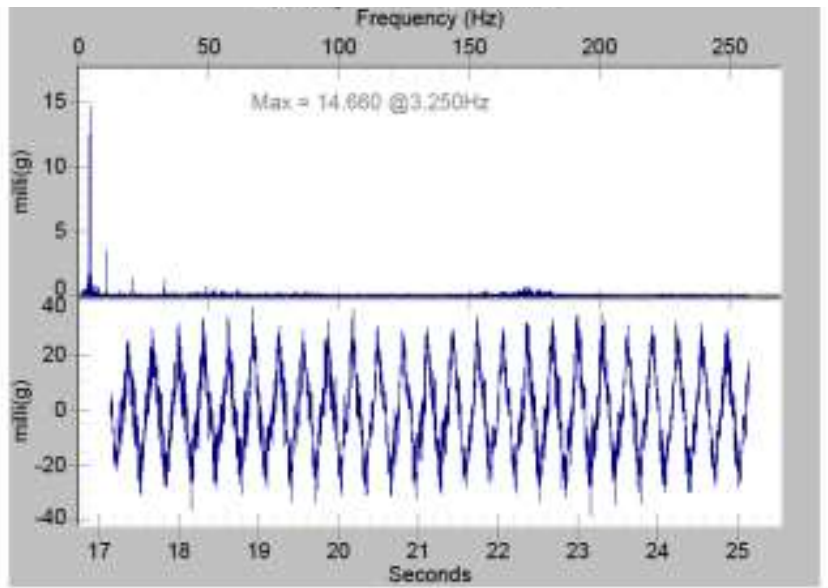

Figure 4. FFT frequency spectrum

- The vertical (bounce) vibrations are transmitted to the car through the suspension rope system which can be explained using the spring-mass model shown in Fig. 5 where $K_{e}$ represents the effective stiffness coefficient of the suspension ropes, $M$ is the mass of the lift car assembly, $s(t)$ represents the motion excitation at the traction sheave end due to an eccentricity or out-of-roundness error and $x(t)$ is the response / displacement of the car assembly.

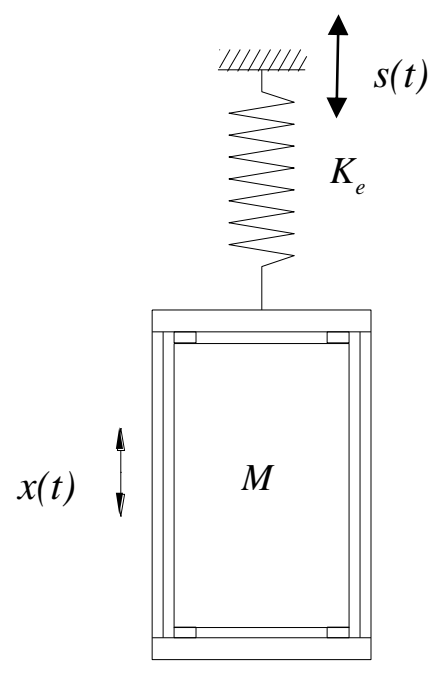

Figure 5 - Spring-mass model

The natural (resonance) frequency of the car-suspension system can be calculated using the following well known equation [4] as 


$$
\omega=\sqrt{\frac{K_{e}}{M}} \frac{\mathrm{rad}}{s}\left(f=\frac{\omega}{2 \pi} \mathrm{Hz}\right)
$$

The response can then be determined by solving the following differential equation of motion [9]

$$
\ddot{x}+2 \zeta \omega \dot{x}+\omega^{2} x=s_{\max } \sqrt{\omega^{4}+4(\zeta \omega \Omega)^{2}} \sin \left(\Omega t+\theta_{0}\right)
$$

where the overdots denote the time derivatives, $s_{\max }$ is the maximum displacement due to the excitation at the sheave, $\Omega$ represents the frequency of the pulley, $\zeta$ is the damping ratio. The maximum displacement of the car is then determined as

$$
x_{\max }=s_{\max } \sqrt{\frac{1+4 \zeta^{2} r^{2}}{\left(1-r^{2}\right)^{2}+4 \zeta^{2} r^{2}}}
$$

where $r$ represents the frequency ratio. The corresponding acceleration amplitude is then given as

$$
a_{\max }=s_{\max } \Omega^{2} \sqrt{\frac{1+4 \zeta^{2} r^{2}}{\left(1-r^{2}\right)^{2}+4 \zeta^{2} r^{2}}}
$$

The natural frequency changes during the lift travel and has been determined from the braking tests as $3.125 \mathrm{~Hz}$ at the bottom landing and $4 \mathrm{~Hz}$ at the top landing, respectively (please see Fig. 6 and 7).
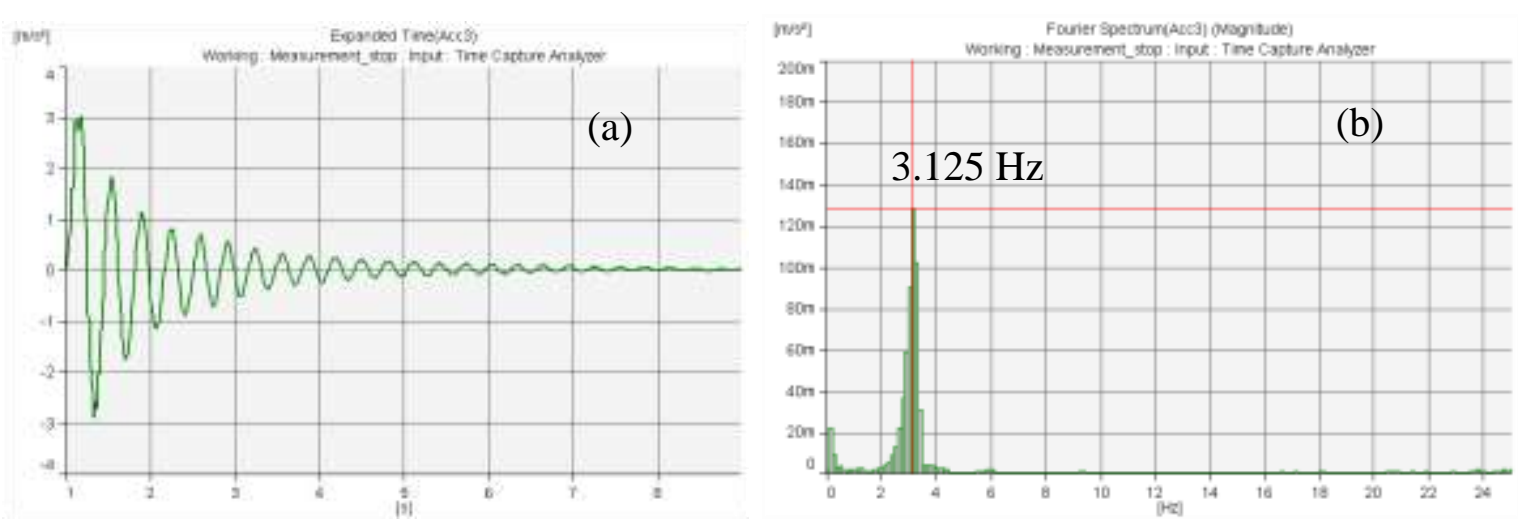

Figure 6. Vibrations measured at the car sling after the brake was applied when approaching the bottom landing (a) time response; (b) Fourier frequency spectrum.

- It is evident that the range of resonance frequencies is close to the rotational frequencies of the diverter pulleys. 

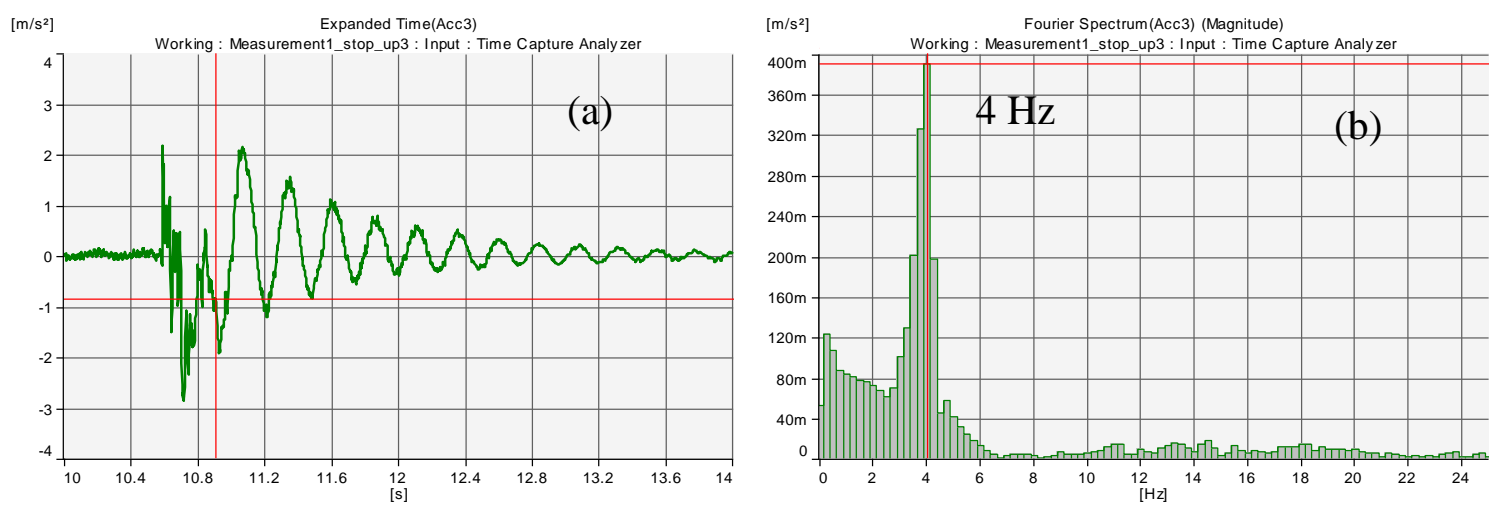

Figure 7. Vibrations measured at the car sling after the brake was applied when approaching the top landing (a) time response; (b) Fourier frequency spectrum.

In these conditions, if the empty car mass is $15,800 \mathrm{~kg}$ and the rated load is $9,100 \mathrm{~kg}$, the suspension stiffness coefficient, with the car approaching the bottom landing is about $K_{e}=10.545 \mathrm{MN} / \mathrm{m}$, the natural frequency calculated according to equation (3) is then found to be $3.275 \mathrm{~Hz}$. Assuming that the out-roundness error results in the maximum displacement $s_{\max }=0.1 \mathrm{~mm}$, with a small damping ratio of $1 \%$, the maximum displacements of the fully loaded car calculated from equation (5) may reach $2.5 \mathrm{~mm}$ and the corresponding acceleration determined from equation (6) will be over 100 milli-g. Thus, it is evident from the results above that the dynamic interactions between the pulleys, the car suspension ropes and the car are responsible for compromising the ride quality of the system.

\section{HOW TO IMPROVE NOISE AND VIBRATION PERFORMANCE}

The following three basic principles can be applied to mitigate the effects of / reduce noise emission and vibration in mechanical systems:

1. Prevention. To reduce the strength of the source.

2. De-coupling. To interrupt the noise/vibration path.

3. Damping. To absorb the energy of noise/vibration.

However in lift systems, due to their unique design and principles of operation, not all available measures can be applied when it comes to the issue of noise and vibration mitigation.

\section{CONCLUSION}

The assessment of influence of noise and vibration in a PTS should take into account the subjective perception of passengers. However, there is limited evidence of how this influence can be quantified. But, the causes of noise and vibration and their effects (dynamic responses) can be quantified. If the excitation forces are identified the responses are determined through the application of experimental techniques and/or calculated using analytical techniques and/ or computer simulation methods. Relevant mitigation measures to reduce their effect can then be applied.

The key dynamic parameters that influence a PTS it can be identified as follows.

- PTS ride quality can be assessed by the introduction of certain thresholds, such as the maximum acceleration, jerk and/ or noise levels. However, common limits of these values have not been agreed, as they strongly relate to market segments or country-specific acceptance levels.

- Noise and vibration can cause multiple, possibly severe damage in human physiological sub-systems. However, the human response to noise and vibration is as diverse as humankind is and in most cases uncritical in PTS. However, in some cases excessive vibration might lead to a failure of the design components and compromise passenger safety. 
- PTS ride quality is affected by various sources of excitation that are related to elements such as rails, rail joints, roller guides, imperfect rotating components or ropes.

- The most critical phenomenon is external resonance (forced vibration due an excitation originating from an external source), as this often leads to an instability of the vibrating system.

- Active and passive measures can be applied to mitigate the effects of resonance, noise and vibration.

\section{REFERENCES}

[1] R. E. Howkins, Elevator Ride Quality - the Human Ride Experience. In: Proceedings of Elevcon 2006, 20 - 22 June 2006, Helsinki, Finland, 100-110.

[2] R. Smith, Achieving Good Ride Quality. In: Proceedings of the Symposium on the Mechanics of Slender Structure (MoSS 2006), Northampton, UK, 28 - 29 September 2006 (on CD ROM).

[3] ISO 18738-1, Measurement of ride quality, Part 1: Lifts/Elevators (2012)

[4] S. S. Rao, Mechanical Vibrations SI Edition. Prentice Hall, Singapore (2005)

[5] M. J. Griffin, Handbook of Human Vibration. Academic Press Limited, London (1990)

[6] I. Herrera, and S. Kaczmarczyk, The Assessment of Vibration Absorption Capacity of Elevator's Passengers. Journal of Physics: Conference Series, Vol. 181, 2009, 012081

[7] P. Feldhusen, Simulation Method for Vibration Control System on High Rise Elevators: Feasibility Study. Internal Report, ThyssenKrupp Elevator AG, December 14, 2013.

[8] J.P. Andrew, and S. Kaczmarczyk, Systems Engineering of Elevators. Elevator World, Inc., Mobile, Alabama (2011).

[9] S. Kaczmarczyk, Vibration Problems in Lift and Escalator Systems: Analysis Techniques and Mitigation Strategies. In: Proceedings of the $3^{\text {rd }}$ Symposium on Lift and Escalator Technologies, Northampton, UK, 26-27 September 2013, 71-79.

[10] G. R. Strakosch, The Vertical Transportation Handbook, John Wiley, New York, 1998.

[11] M. Iida, Y. Sakuma, Comfort of Ultra-high Speed Elevators. Mitsubishi Electric Advance, Vol. 144, 2013, pp. 2-5.

[12] S. Kaczmarczyk, The Prediction and Analysis of Lift Car - Hoist Rope Vibration Interactions. In: Proceedings of ELEVCON 2005, 7-9 June 2005, Beijing, China, 108-117.

[13] J.P. Andrew, and S. Kaczmarczyk, Rope Dynamics. Elevator World, July 2011, 45-56.

[14] S. Kaczmarczyk, J.P. Andrew, The Modelling and Prediction of Non-stationary Vibrations in Lift Systems. Tecnologia del Ascensor, Actas del ELEVCON 2003, Barcelona, Spain, March 2003, 107-117.

[15] G.X. Shen, H.L. Bai, A.T.P. So, Experiments on Aerodynamics of Super High Speed Elevators. In: Proceedings of ELEVCON 2005, 7-9 June 2005, Beijing, China, 174-184. 
[16] K. Funai, H. Katayama, J. I. Higaki, K. Utsonomiya, S. Nakashima, The Development of Active Vibration Damper for Super High-Speed Elevators. In: Proceedings of ELEVCON 2004, 27-29 April 2004, Istanbul, Turkey, 81-89.

[17] R. Sánchez Crespo, S. Kaczmarczyk, P. Picton, H. Su, M. Jetter, Modelling and Simulation of a high-rise elevator system to predict the dynamic interactions between its components. Proceedings of the $3^{\text {rd }}$ Symposium on Lift and Escalator Technologies, Northampton, UK, 26-27 September 2013, 43-52.

[18] C. Coffen, L. Hardin, T. Derwinski, Statistical Energy Analysis of a High Speed Elevator Cab and Frame. In: Proceedings of the $5^{\text {th }}$ International Congress on Sound and Vibration, December 15-18, 1997, Adelaide, South Australia, 2295-2303. 